\title{
De la videopolítica a la ciberpolítica: debate entre candidatos y televisiones en cinco elecciones presidenciales
}

\section{From video politics to cyberpolitics: Debate among candidates and televisions in five presidential elections}

\author{
Paulo-Carlos López-López; Pablo Oñate
}

Cómo citar este artículo:

López-López, Paulo-Carlos; Oñate, Pablo (2019). “De la videopolítica a la ciberpolítica: debate entre candidatos y televisiones en cinco elecciones presidenciales". El profesional de la información, v. 28, n. 5, e280512. https://doi.org/10.3145/epi.2019.sep.12

Artículo recibido el 25-04-2019

Aceptación definitiva: 11-09-2019
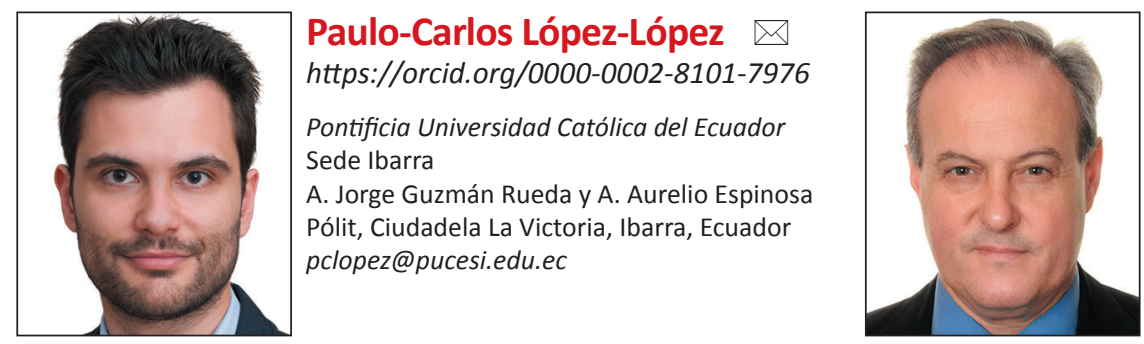

Pablo Oñate

https://orcid.org/0000-0003-4362-3083

Universidad de Valencia

Departamento de Derecho Constitucional,

Ciencia Política y Administración

Avda. de los Naranjos, $\mathrm{s} / \mathrm{n}$.

46022 Valencia, España

pablo.onate@uv.es

\section{Resumen}

Los medios de comunicación se convierten en actores políticos también en el espacio digital, en dónde su información construye una agenda que se relaciona con candidatos y ciudadanos. La presente investigación analiza las cuentas oficiales de Twitter de las dos principales televisiones en Argentina, Perú, Ecuador, Honduras y Chile durante 28 días, que corresponden con los procesos electorales y campañas de las elecciones presidenciales celebradas en América Latina en el período 2015-2017. A través del estudio de 16.359 emisiones mediante herramientas como Python Twint, NVivo y SPSS, se correlacionan las temáticas más relevantes en cada proceso electoral, poniéndolas en discusión con las agendas de los respectivos candidatos. Como principales conclusiones, se indica la primacía del horse-race campaign, la posición subsidiaria de la información política y la existencia de una "coalición de agendas" que marca la relación entre el sistema político y el sistema mediático.

\section{Palabras clave}

Twitter; Agenda-setting; América Latina; Elecciones; Comunicación política; Información política; Redes sociales.

\begin{abstract}
Media acquires political prominence also in the digital space, where its information collaborates in building up an agenda linked to both candidates and citizens. The present investigation analyzes the official Twitter accounts of two main television stations in Argentina, Peru, Ecuador, Honduras and Chile for 28 days, each of which corresponds with the electoral processes and campaigns of the presidential elections held in Latin America over the period 2015-2017. Through the study of 16,359 broadcasts using tools such as Python Twint, NVivo and SPSS, the most relevant topics from each electoral process were correlated and analyzed in relation to the agendas of the candidates. The main conclusions show the primacy of the horse-race campaign, the subsidiary position of political information and the existence of a "coalition of agendas" that marks the relationship between the political system and the media system.
\end{abstract}

Financiación

Los resultados de este artículo corresponden al proyecto DEBATV, Debates Electorales Televisados en España: Modelos, Proceso, Diagnostico y Propuesta (CSO2017-83159-R), proyecto de I+D+I (Retos) financiado por el Ministerio de Economía, Industria y Competitividad (Mineco) y la Agencia Estatal de Investigación (AEI) del Gobierno de España, con el apoyo del Fondo Europeo de Desarrollo Regional (Feder) de la Unión Europea (UE). Igualmente ha colaborado técnicamente la Universidad de Palermo (Buenos Aires, Argentina). 


\section{Keywords}

Twitter; Agenda-setting; Latin America; Elections; Political communication; Political information; Social media.

\section{Introducción y estado de la cuestión}

Para los medios de comunicación -y por consiguiente para las televisiones-, la configuración de un nuevo espacio de competición en el ámbito digital, principalmente a través de las llamadas redes o medios sociales, ha propiciado emergentes dinámicas de interacción con partidos políticos y ciudadanos (Wolton, 1997). Esa interacción tiene una doble manifestación en la Red: por un lado, a través de sus respectivas estrategias partidarias y mediáticas en lo que se refiere a la producción de contenidos y mediación; por otro, en los mecanismos reales de participación y debate propios del ciudadano-elector. No obstante, las investigaciones de los últimos años han demostrado que los partidos políticos hacen un uso superficial, ineficiente y muy intuitivo de esa interacción comunicativa en las redes (Cárdenas; Ballesteros; Jara, 2017), y ello pese a que en la audiencia se hayan detectado nuevas formas de construcción de la agenda pública (Casero-Ripollés, 2015).

Cuando los medios de comunicación diseñan la agenda mediática digital tienen en cuenta un conjunto de factores: los flujos del mercado publicitario, las pautas de actuación de las cuentas oficiales y de sus periodistas en Twitter para mantener la reputación digital (Noguera-Vivo, 2010), así como las pautas de producción de la información.

De forma complementaria, hay que considerar dos reflexiones sobre las televisiones y sobre el ejercicio del periodismo político en la Red:

- en primer lugar, si existe la denominada cobertura desmedida del asunto político, que estaría reforzando la tendencia de dar entrada a todo lo que nace por iniciativa de un partido;

- en segundo lugar, si la pulsión de control que se realiza en el ámbito convencional por parte de los partidos, sobre todo en campaña electoral (Rabadán, 2015), se traslada a la agenda digital a través de la tematización y el encuadre, es decir, priorizando unos temas sobre otros y estableciendo los marcos de interpretación de los mismos.

\subsection{Poder político y medios de comunicación}

La relación entre el poder político y los medios de comunicación -bien sea a través de su relación desde el punto de vista estructural (Hallin; Mancini, 2009) o a través de cómo se plasma en distintas prácticas- ha sido ya profusamente estudiada (Larsson, 2002; Cohen; Tsfati; Sheafer, 2008; Castells, 2008; Casero-Ripollés, 2009; Ekman; Widholm, 2015). En muchas ocasiones esa relación se caracteriza por el control político y gubernamental de los medios (Soengas-Pérez, 2015), manifestada a través de mecanismos formales (leyes, concesiones y control legislativo), económicos (subvenciones y publicidad institucional) o del propio ejercicio de la producción periodística (fuentes, organización de actos, filtraciones o presiones), a lo que se suma la eventual dependencia que genera la intromisión en el quehacer profesional y en la libertad de acción de los periodistas, también en América Latina (Segado-Boj; Díaz-Campo; Lloves-Sobrado, 2015). En el caso de las radiotelevisiones públicas, también se da esa intromisión en la designación de órganos de dirección, de gestión y de asesoramiento (De-Miguel; Pozas, 2009).

Esta relación entre poder político y mediático afecta a la calidad democrática y, en consecuencia, al desarrollo de un país. A las funciones clásicas de socialización política, armonización de intereses, y canalización de las demandas del ciudadano-elector al sistema por parte de los partidos, se le suma la que desempeñan los medios de comunicación en la sociedad de convergencia tecnológica: la alfabetización digital o la discusión pública, que es efectiva en tanto que el ejercicio del periodismo político es independiente.

Se pueden destacar cuatro características de las dinámicas de esa relación en el contexto latinoamericano (Becerra, 2014):

1) ausencia de una concepción de servicio público en los medios privados y una utilización de los medios públicos para difundir el discurso del gobierno de turno;

2) concentración empresarial en el ámbito de la comunicación -muy articulada con el poder político-como una de las mayores trabas para que los medios fomenten una democracia de más calidad, y en donde la diversidad debe ser algo más que la existencia de una propiedad no oligopólica, lo que debe manifestarse también en verdadera multiplicidad de los contenidos (Mastrini; Becerra, 2007);

3) marcada configuración centro-periferia del propio Estado, generando contenidos desde la centralidad urbana y obviando al resto del territorio, que se convierte en mero consumidor;

4) sistema mediático poco regulado pero muy controlado a través de la relación entre los gobiernos y los dueños de las empresas (Fox; Waisbord, 2002). 


\subsection{De la videopolítica a la ciberpolítica}

En las estrategias de difusión del mensaje político a través de los medios de comunicación, el último tercio del siglo XX presenció la preponderancia de la televisión sobre el resto de los medios masivos, principalmente la prensa y radio, convirtiéndose en el espacio central de la confrontación electoral. Ello se basó en la construcción de las características de la videopolítica: su incidencia en los procesos políticos mediante una transformación de su concepción (Sartori, 1998) a través de la mediatización, la audiovisualización, la espectacularización y la personalización (Martínez-Pandiani, 2006). No obstante, la irrupción en la última década del nuevo ecosistema digital de carácter híbrido (Chadwick, 2013) ha propiciado un nuevo escenario y profundas transformaciones, no sólo tecnológicas, sino también organizativas. Los partidos deben tener en cuenta el proceso de convergencia periodística (Salaverría; Avilés, 2008), que afecta al ámbito tecnológico, empresarial y editorial de los medios, a lo que hay que sumar un relato transmediático (López-López; Romero-Ortega; Ramos-Gil, 2018) que, si bien modifica parcialmente la adaptación del mensaje a los públicos que consumen los medios digitales, no altera -aparentemente- el contenido clásico (López-López; Vásquez-González, 2018).

De esta forma se ha pasado de la llamada videopolítica, como elemento organizador de la agenda, a otras formas de representación, como la política 2.0 o tecnopolítica (Baggiolini; Castro-Rojas, 2016), una forma de apropiación y uso de las plataformas como base para el debate -o los debates-y la confrontación, muy especialmente electoral. La tecnopolítica se inserta dentro de un concepto mucho más general como es el de la ciberpolítica, entendida desde el punto de vista de la interacción entre gobernantes y gobernados, como también una expresión de un

"sueño ciberdemocrático frente a una politicidad ilustrada y representativa que se exhibe públicamente insuficiente, desbordada y abierta a la crisis (...) lo que permite a la gente exigir a las instituciones su inmersión en un proceso de radicalización democrática" (Erro-Sala, 2015, p. 147).

Las investigaciones sobre videopolítica, además de centrarse en su caracterización y efectos (Sánchez-Galicia, 2018), han avanzado en la relación que establece con el concepto de democracia (Acinas-Vázquez, 2009) o mediocracia (Trejo-Delarbre, 1994), así como en la transición que ha supuesto la aparición de las tecnologías de la información y del conocimiento, del "homo Videns al homo Twitter" (Cansino; Calles-Santillana; Echeverría, 2016, p. 1). Esto genera un punto de partida teórico para el análisis de la ciberpolítica desde aproximaciones críticas (Rendueles; Sádaba, 2014), además del estudio de los microrrelatos (Baggiolini; Castro-Rojas, 2016) o la creación de la política relatada mediante el storytelling (López-Hermida-Russo; Monardes, 2013).

En este contexto -más allá de una modificación instrumental-, también existe una revisión epistemológica de teorías como la de la agenda-setting (McCombs; Shaw, 1972): ya no se puede hablar de una agenda pública, sino de varias agendas públicas a través de un modelo de fusión (Weaver; McCombs; Shaw, 2004) caracterizado por la existencia de ciberguetos; igualmente, el rol de los medios estaría en cierta medida bloqueado por la llamada "burbuja de filtros" (Pariser, 2011), que establece una relación con la audiencia mediada a través de algoritmos; igualmente, la transmisión de información se realizaría a través de paquetes, que fijan en la mente de la ciudadanía una serie de atributos, indicando no solamente el tema sobre el cual pensar, sino también qué asociar y cómo realizarlo (Vu; Guo; McCombs, 2014). Por ello, la llamada coalición de agendas entre partidos y medios para incidir en la opinión pública parece haberse difuminado parcialmente, cambiando su ejercicio de mediación o hipermediaciones (Scolari, 2008), pero en ningún caso desapareciendo; simplemente mutando y complementándose, con un contenido político en el que sigue primando el horse-race campaign frente al issue-campaign (López-López; Oñate; Chavero Ramírez, 2018), es decir, constituyendo una plataforma para el diálogo cruzado en lugar de la construcción de un espacio de propuestas. Por todo ello, resulta relevante indagar acerca de las relaciones que se operan en la construcción de la agenda política y su evolución en los procesos electorales competitivos.

\section{Metodología y datos}

\subsection{Pregunta de investigación, hipótesis y objetivos}

El presente trabajo continúa las investigaciones sobre la caracterización de la agenda política digital en América Latina (López-López; Oñate; Chavero-Ramírez, 2018), el análisis de la presencia de las televisiones en la confrontación electoral (Fenoll-Tomé; Cárcamo-Ulloa; Sáez-Trumper, 2018), y sus temas (López-López, Vásquez-González, 2018). Para ello, en estas páginas nos centraremos en el análisis de la información publicada en Twitter por las televisiones de Argentina, Perú, Ecuador, Honduras y Chile en respectivos períodos electorales. El método utilizado ha sido el analítico, aplicando una metodología mixta a través de una técnica de análisis de contenido y del discurso. El proceso de extracción de los datos se ha realizado con la librería de Python Twint*, creando un script que contuviera las fechas de inicio y final de la observación.

*Twitter Intelligence Tool

https://github.com/haccer/twint 
La información se procesó mediante el software cualitativo Nvivo, previo al análisis manual de cada uno de los tweets, la creación de las distintas agendas y realizando los cruces mediante SPSS, aplicando el coeficiente de correlación Rho de Spearman. El objetivo principal del estudio es analizar la agenda mediática digital en relación con las estrategias partidarias en las elecciones presidenciales de América Latina en el período 2015-2017, formulando la siguiente pregunta de investigación:

¿En qué medida los mensajes de las televisiones han sido similares a los de los candidatos en los procesos electorales presidenciales?

Como aportación a la teoría, se pretende verificar o refutar el modelo de "coalición de agendas" entre medios y partidos para influir en la opinión pública (Chavero et al., 2013), poniendo en discusión nuestros resultados con trabajos precedentes. Sobre esta base, se parte de dos hipótesis principales y dos secundarias:

- H1. Existe una relación temática estadísticamente relevante entre los mensajes de los medios de comunicación públicos y los de los candidatos oficialistas, además de un claro encuadre positivo en favor de los últimos.

- H2. Los medios de comunicación privados tienden a construir agendas más similares con los candidatos de la oposición.

- h1. En el período electoral la información política ocupa una posición subsidiaria en las televisiones analizadas.

- h2. Los medios de comunicación privados promueven una agenda temática similar en el ámbito regional, con una presencia destacada de la política internacional y la institucionalidad.

Las correlaciones (entre -1 y 1) se calculan con la agenda temática de las televisiones, que se discute con la información emitida en Twitter en el mismo período electoral por parte de los candidatos, ya estudiada en una investigación precedente (López-López; Vásquez-González, 2018). Por su parte, la codificación de los encuadres se ha realizado a través del análisis de los atributos del candidato en cada mensaje, siendo el resultado positivo (+), negativo (-), o neutro (=), con un número final $(\mathrm{N})$ que se halla a través de una simple suma o resta.

Para el presente estudio se han seleccionado, dentro de los medios de comunicación, las televisiones generalistas, siguiendo la teoría de la agenda-setting en su versión más actual (McCombs; Shaw; Weaver, 2014), que incide en que cuanto más próximas están las elecciones, la televisión es el medio que más promueve la enfatización (spotlighting function) y el encuadre de los temas.

Tabla 1. Objeto de estudio

\begin{tabular}{|c|c|c|c|c|}
\hline País & Fecha comicios & Período analizado & Cuentas de televisiones & Número de emisiones \\
\hline \multirow{2}{*}{ Argentina } & \multirow{2}{*}{22 de noviembre de 2015} & \multirow{2}{*}{$\begin{array}{l}\text { Del } 23 \text { de octubre al } 20 \text { de } \\
\text { noviembre }\end{array}$} & $\begin{array}{l}\text { TV Pública Argentina } \\
\text { (@tv_publica) }\end{array}$ & 2.053 \\
\hline & & & $\begin{array}{l}\text { TV Federal } \\
\text { (@telefenoticias) }\end{array}$ & 1.709 \\
\hline \multirow{2}{*}{ Perú } & \multirow{2}{*}{5 de junio de 2016} & \multirow{2}{*}{$\begin{array}{l}\text { Del } 6 \text { de mayo al } 3 \text { de junio } \\
\text { de } 2016\end{array}$} & $\begin{array}{l}\text { TV Perú } \\
\text { (@noticias_tvperu) }\end{array}$ & 1.214 \\
\hline & & & $\begin{array}{l}\text { Latina Televisión } \\
\text { (@Latina_pe) }\end{array}$ & 2.630 \\
\hline \multirow{2}{*}{ Ecuador } & \multirow{2}{*}{2 de abril de 2017} & \multirow{2}{*}{$\begin{array}{l}\text { Del } 3 \text { al } 31 \text { de marzo de } \\
2017\end{array}$} & $\begin{array}{l}\text { EcuadorTV } \\
\text { (@EcuadorTV) }\end{array}$ & 1.523 \\
\hline & & & $\begin{array}{l}\text { Ecuavisa } \\
\text { (@ecuavisa) }\end{array}$ & 2.261 \\
\hline \multirow{2}{*}{ Honduras } & \multirow{2}{*}{26 de noviembre de 2017} & \multirow{2}{*}{$\begin{array}{l}\text { Del } 27 \text { de octubre al } 24 \text { de } \\
\text { noviembre de } 2017\end{array}$} & $\begin{array}{l}\text { TNH } \\
\text { (@TNH_canal8) }\end{array}$ & 706 \\
\hline & & & $\begin{array}{l}\text { Televicentro HN } \\
\text { (@televicentrohn) }\end{array}$ & 1.961 \\
\hline \multirow{2}{*}{ Chile } & \multirow{2}{*}{17 de diciembre de 2017} & \multirow{2}{*}{$\begin{array}{l}\text { Del } 17 \text { de noviembre al } 15 \\
\text { de diciembre de } 2017\end{array}$} & $\begin{array}{l}\text { TVN } \\
\text { (@tvn) }\end{array}$ & 1.521 \\
\hline & & & $\begin{array}{l}\text { Canal 13 } \\
\text { (@canal13) }\end{array}$ & 781 \\
\hline \multicolumn{4}{|l|}{ TOTAL } & 16.359 \\
\hline
\end{tabular}

Debido a la configuración de los sistemas mediáticos en América Latina, dentro de las televisiones se ha entendido relevante su selección en función de su titularidad, eligiendo una pública, además de una privada, en atención a su audiencia analógica (Berrendo-Pérez; Serrano-Fernández; Encinas-Puebla, 2019) y su impacto (número de seguidores) a través de Twitter. Las televisiones analizadas, junto con los respectivos candidatos, son las siguientes:

\section{Argentina}

Televisión Pública Argentina (@tv_publica), Televisión Federal (@telefenoticias)

Mauricio Macri (@mauriciomacri) y Daniel Scioli (@danielscioli). 
Perú

TV Perú (@tnoticias_tvperu), Latina Televisión (@Latina_pe)

Pedro Pablo Kuczynski (@ppkamigo) y Keiko Fujimori (@KeikoFujimori).

\section{Ecuador}

EcuadorTV (@EcuadorTV), Ecuavisa (@ecuavisa)

Lenín Moreno (@Lenin)y Guillermo Lasso (@LassoGuillermo).

\section{Honduras}

TNH (@TNH_canal8), TelevicentroHN (@televicentrohn)

Juan Orlando Hernández (@JuanOrlandoH) y Salvador Nasralla (@SalvadorAlianza).

\section{Chile}

TVN (@tvn), Canal 13 (@canal13)

Sebastián Piñera (@sebastianpinera) y Alejandro Guillier (@guillier).

En el caso de las televisiones se han descargado las emisiones efectuadas en los perfiles generalistas las últimas cuatro semanas antes del proceso electoral de cada país (Argentina, Perú Ecuador, Honduras y Chile) entre los años 2015 y 2017, con un total de 16.362 emisiones y 65.448 categorías de análisis. Se han excluido los procesos electorales en Haití (por los sucesivos procesos de impugnación), y Nicaragua y Guatemala (por no registrarse una agenda política relevante -pocas emisiones o inexistencia de estrategias comunicativas online-).

\section{Resultados}

\subsection{Elecciones en Argentina}

22 de noviembre de 2015

Las elecciones argentinas, analizadas del 23 de octubre al 20 de noviembre de 2015, supusieron el fin de ciclo del Kirchnerismo al no poder presentarse Cristina Fernández de Kirchner a la reelección. No obstante, con su aval concurriría el oficialista Daniel Scioli, que se enfrentaría al candidato conservador Mauricio Macri. El primero tendría en la Televisión Pública Argentina un claro encuadre positivo (relación de 2:1 en frecuencia con más de una treintena de encuadres positivos) con un componente ideológico muy marcado, pero sin correspondencia temática por la aparición constante de la ex presidenta, acompañándolo como segunda voz y con mayor peso que el candidato oficial, lo que acabó distorsionando el rango. Por su parte, la presencia de Mauricio Macri, muy escasa, estuvo acompañada siempre de encuadres negativos ("Mira como Macri disfruta de sus vacaciones en plena campaña") y de continuas referencias a las "calamidades" de su modelo de política económica ejemplificado en el Área de Libre Comercio para las Américas (ALCA) (con un especial sobre la conmemoración del "No" argentino, pobreza, reducción de derechos laborales y sociales).

Tabla 2. Agenda, correlaciones y encuadres en Argentina

\begin{tabular}{|c|c|c|c|c|c|c|c|c|c|c|}
\hline \multicolumn{3}{|c|}{ Agenda TV Pública } & \multicolumn{5}{|c|}{ Correlaciones y encuadres } & \multicolumn{3}{|c|}{ Agenda Telefe } \\
\hline & & & \multicolumn{5}{|c|}{ Cálculo coeficiente correlación Spearman } & \multirow{3}{*}{ Rango y tema } & \multirow{3}{*}{$\mathrm{N}$} & \multirow{3}{*}{$\%$} \\
\hline Rangovtom? & N & $R^{2}$ & \multirow{3}{*}{ TPA } & TPA & TLF & MAC & SCI & & & \\
\hline Kango y lemd & $\mathrm{N}$ & $\%$ & & 1,000 & 0,538 & 0,559 & 0,420 & & & \\
\hline 1-Campaña & 486 & 52,2 & & 0.538 & 1000 & $0,727^{* *}$ & 0,392 & 1-Campaña & 270 & 60,5 \\
\hline 2-Política internacional & 75 & 8,1 & \multirow{3}{*}{\begin{tabular}{|l|} 
TLF \\
MAC \\
SCI \\
\end{tabular}} & & & \multirow{2}{*}{1,000} & \multirow{2}{*}{$0,783^{* * *}$} & 2-Política económica & 25 & 5,6 \\
\hline 3-Política económica & 69 & 7,4 & & 0,559 & 0,121 & & & 3-Institucionalidad & 22 & 4,9 \\
\hline 4-Institucionalidad & 48 & 5,2 & \multirow{5}{*}{\multicolumn{5}{|c|}{$\begin{array}{l}\text { ** La correlación es significativa al nivel } 0,01 \\
\text { (bilateral). } \\
\text { Existe una coalición de agendas entre la televisión } \\
\text { privada y el candidato Mauricio Macri }(0,727) \text {. } \\
\text { Frecuencia de mención de candidatos }\end{array}$}} & 4-Colectivos prioritarios & 20 & 4,5 \\
\hline 5-Ideología & 46 & 4,9 & & & & & & 5-Seguridad & 18 & 4,0 \\
\hline 6-Empleo & 31 & 3,3 & & & & & & 6-Servicios públicos & 14 & 3,1 \\
\hline 7-Economía e industria & 30 & 3,2 & & & & & & 7-Política internacional & 13 & 2,9 \\
\hline 8-Servicios públicos & 22 & 2,4 & & & & & & 8-Política social & 12 & 2,7 \\
\hline 9-Seguridad & 19 & 2,0 & \multicolumn{2}{|c|}{ TV/Candidato } & \multicolumn{2}{|c|}{ Macri } & Scioli & 9-Ideología & 9 & 2,0 \\
\hline 10-Ciencia & 17 & 1,8 & \multicolumn{2}{|c|}{ TV Pública } & \multicolumn{2}{|c|}{$67,65 \%$} & $32,35 \%$ & 10-Obra pública & 7 & 1,6 \\
\hline 11-Política social & 16 & 1,7 & \multicolumn{2}{|l|}{ Telefe } & 51,05 & & $48,95 \%$ & 11-Ciencia & 6 & 1,3 \\
\hline 12-Otros & 72 & 7,8 & Encuad & de candi & latos & & & 12-Otros & 30 & 6,9 \\
\hline TOTAL & 931 & 100 & TV/C & Ididato & Mac & & Scioli & TOTAL & 446 & 100 \\
\hline & & & TV Púl & & -33 & & +31 & & & \\
\hline & & & Telefe & & +6 & & -10 & & & \\
\hline
\end{tabular}

Fuente: elaborado a partir de observaciones mencionadas en texto 
El ente público ha asumido una posición de parte muy clara con el discurso gubernamental, incluso introduciendo al Papa Francisco en campaña, "ya saben lo que pienso", con una presencia sostenida de la economía (los llamados temas duros de agenda) en sus mensajes, siendo especialmente llamativo cómo en el debate realizado el día 15 de noviembre las redes sociales oficiales ocultaron al opositor. Por su parte, el canal de noticias de Televisión Federal (privado) asume, de partida, una posición mucho más neutral y más sutil, con críticas a través de los encuadres a Scioli (-10) pero con una aparición muy similar por parte de ambos candidatos, realizando un ejercicio de mediatización mucho más claro. En cuanto a las temáticas, si bien la información política está hegemonizada por la intendencia de campaña, existen notables diferencias en la información de las televisiones (sobre todo en el enfoque social y de política internacional), con una correspondencia entre Mauricio Macri y el ente privado (institucionalidad, política económica y seguridad). Cabe destacar la alta presencia de noticias de infoentretenimiento, así como la inclusión de la llamada "memoria histórica" en su programación y la discusión ideológica marcada aún por el llamado peronismo, corriente transversal centrada en la figura de Juan Domingo Perón reclamada por Daniel Scioli.

Las elecciones fueron ganadas por Mauricio Macri.

\subsection{Elecciones en Perú}

5 de junio de 2016

La elección presidencial en Perú de 2016, analizada entre el 6 de mayo y el 3 de junio, estuvo caracterizada por un hecho que no se reproduce, prácticamente, en el resto de los comicios: ni el presidente saliente ni su coalición política (independientemente de a qué candidato apoyaran sus partes constitutivas) participaron de la confrontación electoral. Esto provoca que ni la agenda institucional ni la política tuvieran las mismas prioridades en el período analizado. TV Perú desarrolló una actividad periodística en la Red marcada por la ponderación, la responsabilidad y cierta imparcialidad: mostró encuestas a favor de la candidata populista (que provocó un cierto efecto bandwagon o arrastre en el electorado) pero a la vez la encuadró negativamente, con el tema recurrente de la corrupción y el fraude (rango 3).

Tabla 3. Agenda, correlaciones y encuadres en Perú

\begin{tabular}{|c|c|c|c|c|c|c|c|c|c|c|c|}
\hline \multicolumn{3}{|c|}{ Agenda TV Perú } & \multicolumn{6}{|c|}{ Correlaciones y encuadres } & \multicolumn{3}{|c|}{ Agenda Latina } \\
\hline \multirow[b]{2}{*}{ Rango y tema } & \multirow[b]{2}{*}{$\mathbf{N}$} & \multirow[b]{2}{*}{$\%$} & \multicolumn{6}{|c|}{ Cálculo coeficiente correlación Spearman } & \multirow[b]{2}{*}{ Rango y tema } & \multirow[b]{2}{*}{$\mathbf{N}$} & \multirow[b]{2}{*}{$\%$} \\
\hline & & & & $T V P$ & LAT & \multicolumn{2}{|c|}{ FUJ } & KUZ & & & \\
\hline 1-Campaña & 226 & 35,1 & TVP & 1,000 & $0,776^{* *}$ & \multicolumn{2}{|c|}{0,385} & 0,357 & 1-Campaña & 170 & 50,9 \\
\hline 2-Institucionalidad & 102 & 16,0 & LAT & $0,776^{* *}$ & 1,000 & \multicolumn{2}{|c|}{0,462} & 0,308 & 2-Corrupción & 34 & 10,2 \\
\hline 3-Corrupción & 37 & 5,7 & FUJ & 0,385 & 0,462 & \multicolumn{2}{|c|}{1,000} & 0,413 & 3-Seguridad & 30 & 9,0 \\
\hline 4-Economía e industria & 37 & 5,7 & KUZ & 0,357 & 0,308 & \multicolumn{2}{|c|}{0,413} & 1,000 & 4-Institucionalidad & 19 & 5,7 \\
\hline 5-Obra pública & 36 & 5,6 & \multirow{4}{*}{\multicolumn{6}{|c|}{$\begin{array}{l}\text { ** La correlación es significativa al nivel 0,01 } \\
\text { (bilateral). } \\
\text { Existe una coalición de agendas entre las televisiones. } \\
\text { Frecuencia de mención de candidatos }\end{array}$}} & 5-Política internacional & 12 & 3,6 \\
\hline 6-Seguridad & 34 & 5,3 & & & & & & & 6-Política económica & 11 & 3,3 \\
\hline 7-Servicios públicos & 31 & 4,8 & & & & & & & 7- Ideología & 11 & 3,3 \\
\hline 8-Política económica & 30 & 4,7 & & & & & & & 8-Obra pública & 9 & 2,7 \\
\hline 9-Política internacional & 22 & 3,4 & \multicolumn{2}{|c|}{ TV/Candidato } & \multicolumn{2}{|c|}{ Fujimori } & \multicolumn{2}{|c|}{ Kuczynski } & 9-Economía e industria & 8 & 2,4 \\
\hline 10-Política social & 21 & 3,3 & \multicolumn{2}{|l|}{ TVP } & \multicolumn{2}{|c|}{$47,75 \%$} & \multicolumn{2}{|c|}{$52,25 \%$} & 10- Servicios públicos & 7 & 2,1 \\
\hline 11-Colectivos prioritarios & 10 & 1,6 & \multicolumn{2}{|c|}{ Latina } & \multicolumn{2}{|c|}{$53 \%$} & \multicolumn{2}{|r|}{$47 \%$} & 11-Colectivos prioritarios & 3 & 0,9 \\
\hline 12- Otros & 43 & 6,7 & \multicolumn{6}{|c|}{ Encuadres de candidatos } & 12-Otros & 20 & 6,0 \\
\hline \multirow[t]{3}{*}{ TOTAL } & 644 & 100 & \multicolumn{2}{|c|}{ TV/Candidato } & \multicolumn{2}{|c|}{ Fujimori } & \multicolumn{2}{|c|}{ Kuczynski } & TOTAL & 334 & 100 \\
\hline & & & \multicolumn{2}{|l|}{$T V P$} & \multicolumn{2}{|l|}{-10} & \multicolumn{2}{|r|}{+1} & & & \\
\hline & & & \multicolumn{2}{|c|}{ Latina } & -20 & & & +1 & & & \\
\hline
\end{tabular}

Fuente: elaborado a partir de observaciones mencionadas en texto

Latina Televisión, igualmente, tuvo una línea -de inicio- altamente imparcial, utilizando las encuestas electorales sin una vocación de uso político para, conforme avanza el proceso y llega la segunda vuelta presidencial, agudizar de una forma sutil los encuadres negativos de Keiko Fujimori -centrándolos en casos de corrupción de su familia y del empresario y compañero de partido Joaquín Ramírez-. El tratamiento del candidato de centroderecha Kuczynski en ambas corporaciones fue, de igual forma, muy similar: encuadres en su mayor parte neutros, presencia equilibrada y agenda política-mediática muy centrada en los servicios públicos y en la política internacional. Prueba de esta similitud en el tratamiento lo demuestran los resultados de la correlación estadística, en la cual lo más relevante es el 0,776 de TV Perú y Latina Televisión, con la campaña, la corrupción y la institucionalidad situándose, en ambos casos, en la cuatro primeras posiciones de temas con mayor aparición y un similar encuadre.

La elección fue ganada por Pedro Pablo Kuczynski. 


\subsection{Elecciones en Ecuador}

2 de abril de 2017

La segunda vuelta presidencial en Ecuador, estudiada del 3 al 31 de marzo, enfrentó al oficialista Lenín Moreno con el liberal Guillermo Lasso, en unos comicios marcados por la ausencia de Rafael Correa después de casi una década de "Revolución Ciudadana".

Tabla 4. Agenda, correlaciones y encuadres en Ecuador

\begin{tabular}{|c|c|c|c|c|c|c|c|c|c|c|}
\hline \multicolumn{3}{|c|}{ Agenda Ecuador TV } & \multicolumn{5}{|c|}{ Correlaciones y encuadres } & \multicolumn{3}{|c|}{ Agenda Ecuavisa } \\
\hline & & & \multicolumn{5}{|c|}{ Cálculo coeficiente correlación Spearman } & \multirow[b]{2}{*}{ Rango y tema } & \multirow[b]{2}{*}{$\mathbf{N}$} & \multirow[b]{2}{*}{$\%$} \\
\hline Rango y tema & $\mathbf{N}$ & $\%$ & & ETV & $E C U$ & MOR & LASSO & & & \\
\hline 1-Campaña & 166 & 33,1 & ETV & 1,000 & 0,410 & $0,706^{*}$ & 0,371 & 1-Campaña & 80 & 22,1 \\
\hline 2-Colectivos prioritarios & 43 & 8,7 & ECU & 0,410 & 1,000 & 0,196 & 0,315 & 2-Política internacional & 43 & 11,9 \\
\hline 3-Economía e industria & 42 & 8,5 & MOR & $0,706^{*}$ & 0,196 & 1,000 & $0,522^{*}$ & 3-Política económica & 36 & 9,9 \\
\hline 4-Política económica & 29 & 5,9 & LASSO & 0,371 & 0,315 & $0,522^{*}$ & 1,000 & 4-Institucionalidad & 33 & 9,1 \\
\hline 5- Ideología & 29 & 5,9 & \multirow{4}{*}{\multicolumn{5}{|c|}{$\begin{array}{l}\text { ** La correlación es significativa al nivel } 0,01 \\
\text { (bilateral). } \\
\text { Existe una coalición de agendas entre la televisión } \\
\text { pública y el candidato oficialista }(0,706) \\
\text { Frecuencia de mención de candidatos }\end{array}$}} & 5- Empleo & 26 & 7,2 \\
\hline 6-Servicios públicos & 28 & 5,7 & & & & & & 6-Servicios públicos & 20 & 5,5 \\
\hline 7-Corrupción & 22 & 4,5 & & & & & & 7-Ideología & 19 & 5,2 \\
\hline 8- Empleo & 21 & 4,3 & & & & & & 8-Corrupción & 17 & 4,7 \\
\hline 9-Política internacional & 20 & 4,1 & \multirow{2}{*}{\multicolumn{2}{|c|}{ TV/Candidato }} & Moreno & \multicolumn{2}{|c|}{ Lasso } & 9- Economía e industrias & 15 & 4,1 \\
\hline 10-Política social & 20 & 4,1 & & & $66,66 \%$ & \multicolumn{2}{|c|}{$33,33 \%$} & 10-Colectivos prioritarios & 11 & 3,0 \\
\hline 11-Institucionalidad & 17 & 3,5 & \multicolumn{2}{|c|}{ Ecuavisa } & \multirow[t]{2}{*}{$41,9 \%$} & \multicolumn{2}{|c|}{$58,10 \%$} & 11-Política social & 8 & 2,2 \\
\hline 12-Otros & 55 & 11,7 & \multirow{2}{*}{\multicolumn{5}{|c|}{ Encuadres de candidatos }} & 12-Otros & 54 & 15,2 \\
\hline TOTAL & 492 & 100 & & & & & & TOTAL & 362 & 100 \\
\hline & & & \multicolumn{2}{|c|}{ TV/Candidato } & Moreno & \multicolumn{2}{|c|}{ Lasso } & & & \\
\hline & & & \multicolumn{2}{|c|}{ Ecuador TV } & +44 & \multicolumn{2}{|c|}{-5} & & & \\
\hline & & & \multicolumn{2}{|c|}{ Ecuavisa } & -4 & \multicolumn{2}{|c|}{+5} & & & \\
\hline
\end{tabular}

Fuente: elaborado a partir de observaciones mencionadas en texto

La televisión pública ecuatoriana desarrolló una intensa y nada disimulada actividad a favor del candidato oficialista (44 encuadres positivos en el período estudiado), con una aparición sistemática en entrevistas y seguimiento pormenorizado (una relación 2:1 frente a su contrincante) mediante tres estrategias:

a) información sobre las inauguraciones y obras en período electoral por parte de Rafael Correa;

b) uso político de las encuestas (diferencias de hasta 15 puntos) con el claro objetivo de subrayar la diferencia con las relativas al oponente;

c) búsqueda de un encuadre negativo de Guillermo Lasso (-5), ligándolo con el Feriado Bancario -al que se le dedica varios especiales-, y al fraude (empresas offshore y paraísos fiscales). En cuanto a temas, existe una concordancia estadística $(0,706)$ en los mismos tanto en su dimensión como en el rango, destacando aquellos propios de la campaña, los de carácter social y los de economía productiva (pymes, industria y sector primario).

Por su parte, en Ecuavisa un alto porcentaje de las informaciones tienen que ver con el infoentretenimiento, con una escasa presencia de las noticias de carácter político y electoral (16\%). De las mismas existe un alto componente de política internacional en países de la región, mostrando el "fracaso" de los modelos análogos y aliados de Rafael Correa, como el caso de Brasil o la "herencia" de Cristina Fernández de Kirchner en Argentina. En el ámbito interno, destaca la corrupción asociada a Odebrecht, la dimensión sobre los apoyos que recibe cada candidato (muy equilibrado), las propuestas macroeconómicas de Lasso y las dudas sobre la limpieza del proceso electoral (codificadas en "Institucionalidad"). De una forma mucho más soterrada, el canal privado ha generado información positiva para Lasso, reproduciendo su agenda política sin mediación; igualmente ha encuadrado a Moreno de forma negativa, a lo que hay que sumar la cobertura que se ha hecho de su binomio presidencial, Glass, y del expresidente Correa, ligándolo a hechos, obras o políticas de la gestión anterior.

La elección fue ganada por Lenín Moreno.

Se ha pasado de la llamada videopolítica como elemento organizador de la agenda, a otras formas de representación, como la política 2.0 o tecnopolítica 


\subsection{Elecciones en Honduras}

26 de noviembre de 2017

El proceso electoral hondureño (con un análisis realizado entre el 27 de octubre y 24 de noviembre), se vio condicionado por dos eventos que pudieron desvirtuar su normalidad: una catástrofe por lluvias y la situación del Estatus de Protección Temporal para los hondureños en EUA (codificado en "Política Internacional"). También pudo alterar el proceso el hecho de que uno de los candidatos en liza, Salvador Nasralla, fuera presentador de uno de los canales analizados (Televicentro).

Tabla 5. Agenda, correlaciones y encuadres en Honduras

\begin{tabular}{|c|c|c|c|c|c|c|c|c|c|c|}
\hline \multicolumn{3}{|l|}{ Agenda TNH } & \multicolumn{5}{|c|}{ Correlaciones y encuadres } & \multicolumn{3}{|c|}{ Agenda Televicentro } \\
\hline \multirow[b]{2}{*}{ Rango y tema } & \multirow[b]{2}{*}{$\mathbf{N}$} & \multirow[b]{2}{*}{$\%$} & \multicolumn{5}{|c|}{ Cálculo coeficiente correlación Spearman } & \multirow[b]{2}{*}{ Rango y tema } & \multirow[b]{2}{*}{$\mathbf{N}$} & \multirow[b]{2}{*}{$\%$} \\
\hline & & & & TNH & $T L V$ & HER & NAS & & & \\
\hline 1-Campaña & 36 & 18 & TNH & 1,000 & 0,559 & $0,881^{* *}$ & 0,399 & 1-Campaña & 242 & 40,9 \\
\hline 2-Obra pública & 29 & 14,5 & $T L V$ & 0,559 & 1,000 & 0,503 & $0,741^{* *}$ & 2-Institucionalidad & 81 & 13,7 \\
\hline 3-Empleo & 20 & 10 & HER & $0,881^{* *}$ & $0,503^{*}$ & 1,000 & 0,431 & 3-Política internacional & 59 & 10 \\
\hline 4-Política internacional & 19 & 9,5 & NAS & 0,399 & $0,741^{* *}$ & 0,431 & & 4-Seguridad & 36 & 6,1 \\
\hline 5-Institucionalidad & 19 & 9,5 & \multirow{5}{*}{\multicolumn{5}{|c|}{$\begin{array}{l}* * \text { La correlación es significativa al nivel } 0,01 \\
\text { (bilateral). } \\
\text { Existe una coalición de agendas entre la televisión } \\
\text { pública y el candidato oficialista y entre la televisión } \\
\text { privada y el opositor. } \\
\text { Frecuencia de mención de candidatos }\end{array}$}} & 5-Servicios públicos & 29 & 4,9 \\
\hline 6-Seguridad & 16 & 8 & & & & & & 6-Empleo & 21 & 3,6 \\
\hline 7-Política económica & 14 & 7 & & & & & & 7-Corrupción & 20 & 3,4 \\
\hline 8-Economía e industria & 12 & 6 & & & & & & 8-Política económica & 19 & 3,2 \\
\hline 9-Política social & 10 & 5 & & & & & & 9-Economía e industria & 10 & 3,2 \\
\hline 10-Servicios públicos & 8 & 4 & \multirow{2}{*}{\multicolumn{2}{|c|}{ TV/Candidato }} & \multicolumn{2}{|c|}{ Hernández } & Nasralla & 10-Colectivos prioritarios & 15 & 2,5 \\
\hline 11-Colectivos prioritarios & 6 & 3 & TNH & & \multicolumn{2}{|c|}{$100 \%$} & $0 \%$ & 11-Obra pública & 14 & 2,4 \\
\hline 12-Otros & 11 & 5,5 & \multicolumn{2}{|c|}{ Televicentro } & \multicolumn{2}{|l|}{$29,6 \%$} & $69,3 \%$ & 12-Otros & 36 & 6,1 \\
\hline \multirow[t]{5}{*}{ TOTAL } & 200 & 100 & \multirow{2}{*}{\multicolumn{5}{|c|}{ Encuadres de candidatos }} & TOTAL & 591 & 100 \\
\hline & & & & & & & & & & \\
\hline & & & \multicolumn{2}{|c|}{ TV/Candidato } & \multicolumn{2}{|c|}{ Hernández } & Nasralla & & & \\
\hline & & & \multicolumn{2}{|l|}{ TNH } & \multicolumn{2}{|l|}{+16} & 0 & & & \\
\hline & & & \multicolumn{2}{|c|}{ Televicentro } & -3 & & +6 & & & \\
\hline
\end{tabular}

Fuente: elaborado a partir de observaciones mencionadas en texto

Las noticias emitidas en la red social Twitter del canal privado tienen una gran diversificación temática, con tres características principales:

a) una importante apelación a la institucionalidad y a la información básica sobre el proceso electoral y la campaña, haciendo un constante ejercicio de llamamiento a la participación sin violencia y respetando todas las opiniones, elemento importante en un contexto tan polarizado como el hondureño;

b) generación de una agenda propia sin apenas influencia directa de los candidatos;

c) creación de espacio para los tres candidatos principales: Juan Orlando Hernández $(P N H)$, Salvador Nasralla (AOP), y Luis Orlando Zelaya $(P L H)$, con una sobredimensión del segundo y unos encuadres mayormente positivos ligados al concepto de "democracia" y "paz social".

En el caso de la Televisión Nacional de Honduras, la información emitida, asentada en unas aparentes pautas de producción periodística, tiene una clara dimensión propagandística de Hernández, el presidente en funciones. Existe, por una parte, la promoción de las actuaciones e iniciativas gubernamentales (con especial énfasis en la obra pública, en la seguridad y en la gestión del Temporary Protected Status -TPS- a través de una fingida negociación con los EUA); por la otra, una clara ocultación del proceso electoral y de la oposición, que apenas tiene voz (no existe ninguna referencia a los mismos, ni en sentido positivo ni en sentido negativo) transmitiendo un pluralismo político muy limitado, impropio de una televisión pública en un régimen democrático.

En política internacional e institucionalidad, los medios comunicación privados promueven una agenda temática similar en el ámbito regional

La elección fue ganada por Juan Orlando Hernández. 


\subsection{Elecciones en Chile}

17 de diciembre de 2017

La estabilidad del sistema político chileno marca los elencos temáticos, frecuencias y encuadres de los cuatro actores analizados entre el 17 de noviembre y el 15 de diciembre de 2017. En unos comicios que enfrentaron en segunda vuelta al expresidente Sebastián Piñera y al progresista Alejandro Guillier (Michelle Bachelet renunció a presentarse), una de las características comunes de las dos televisiones chilenas en su perfil generalista es el escaso espacio otorgado a la información puramente política y electoral, en detrimento de otro tipo de información propia (autopromoción). Destacaron, en ambos casos, dos programas muy concretos: Las caras de la moneda en Canal 13, dónde se entrevistó con un perfil muy personal a los candidatos Piñera y Guillier, con un formato que dejaba poco espacio para la confrontación de propuestas; y, en la Televisión Pública, el \#DebateAnatel, último cara a cara en el llamado "balotaje" (segunda vuelta presidencial). El número de emisiones relacionadas con los comicios se intensifica en los días previos y posteriores a los propios comicios, muy volcados en la información de servicio público (¿cómo votar?, ¿cuándo?; ¿dónde?), existiendo algunas jornadas en las cuales no existe ningún tipo de tweet ni referencia al proceso electoral. En general, tanto en el ente público con en el privado, los encuadres fueron bastante neutros, las temáticas diversificadas, y se dio una presencia análoga de ambos candidatos, un perfil bajo y una línea informativa que no manifestaba favoritismos evidentes. En lo que se refiere a la producción de la información, abundó el periodismo de declaraciones y los entrecomillados, que permiten la traslación automática de la agenda política a la mediática. En el rango temático el tema "campaña electoral" (53,8\% para TVN; 43,7\% para C13) recogió en ambos casos los actos partidarios, no más allá de describir el lugar en dónde se produjeron los mismos y una posición muy concreta de cada uno de los contendientes sobre algún asunto político determinado. Las altas correlaciones de las informaciones de las televisiones y de los candidatos muestran una campaña tranquila, con temas pactados, muy especialmente los sociales (colectivos prioritarios, política social y servicios públicos), baja conflictividad en política económica, una situación neutra respecto de la seguridad y una ausencia de referencias a la corrupción y el fraude.

Tabla 6. Agenda, correlaciones y encuadres en Chile

\begin{tabular}{|c|c|c|c|c|c|c|c|c|c|c|}
\hline \multicolumn{3}{|c|}{ Agenda TVN } & \multicolumn{5}{|c|}{ Correlaciones y encuadres } & \multicolumn{3}{|c|}{ Agenda Canal 13} \\
\hline & & & \multicolumn{5}{|c|}{ Cálculo coeficiente correlación Spearman } & \multirow[b]{2}{*}{ Rango y tema } & \multirow[b]{2}{*}{$\mathbf{N}$} & \multirow[b]{2}{*}{$\%$} \\
\hline Rango y tema & $\mathbf{N}$ & $\%$ & \multirow{2}{*}{ TVN } & $T V N$ & C13 & PIN & GUI & & & \\
\hline 1-Campaña & 93 & 53,8 & & 1,000 & $0,804^{* *}$ & $0,776^{* *}$ & $0,783^{* *}$ & 1-Campaña & 38 & 43,7 \\
\hline 2-Colectivos prioritarios & 15 & 8,7 & C13 & $0,804^{* *}$ & 1,000 & $0,965^{* *}$ & $0,818^{* *}$ & 2-Ideología & 12 & 13,8 \\
\hline 3-Servicios públicos & 12 & 6,9 & PIÑ & $0,776^{* *}$ & $0,965^{* *}$ & 1,000 & $0,860^{* *}$ & 3-Institucionalidad & 8 & 9,2 \\
\hline 4- Institucionalidad & 9 & 5,2 & & $0,783^{* *}$ & $0,818^{* *}$ & $0,860^{* *}$ & 1,000 & 4-Colectivos prioritarios & 6 & 6,9 \\
\hline 5-Política internacional & 9 & 5,2 & \multirow{4}{*}{\multicolumn{5}{|c|}{$\begin{array}{l}\text { ** La correlación es significativa al nivel 0,01 (bilateral). } \\
\text { Existe una coalición de agendas entre todos los actores } \\
\text { analizados } \\
\text { Frecuencia de mención de candidatos }\end{array}$}} & 5-Servicios públicos & 5 & 5,7 \\
\hline 6-Seguridad & 7 & 4,0 & & & & & & 6-Seguridad & 4 & 4,6 \\
\hline 7-Ideología & 5 & 2,9 & & & & & & 7-Política internacional & 3 & 3,4 \\
\hline 8-Política social & 5 & 2,9 & \multirow{2}{*}{\multicolumn{2}{|c|}{ TV/Candidato }} & \multirow{2}{*}{ Piñera } & \multicolumn{2}{|c|}{ Guillier } & 8-Obra pública & 3 & 3,4 \\
\hline 9-Política económica & 3 & 1,7 & & & & \multicolumn{2}{|c|}{$44,7 \%$} & 9-Economía e industria & 3 & 3,4 \\
\hline 10-Empleo & 3 & 1,7 & \multicolumn{2}{|c|}{ TVN } & $55,3 \%$ & & 年 & 10-Empleo & 2 & 2,3 \\
\hline 11-Economía e industria & 2 & 1,2 & \multicolumn{2}{|l|}{$\mathrm{C} 13$} & 43,070 & & 4,270 & 11-Política social & 2 & 2,3 \\
\hline 12-Otros & 10 & 5,8 & Encuad & de candid & & & & 12-Otros & 1 & 1,3 \\
\hline TOTAL & 173 & 100 & TV/C & didato & Piñera & & uillier & TOTAL & 87 & 100 \\
\hline & & & $T V N$ & & $=$ & & -1 & & & \\
\hline & & & $C 13$ & & -1 & & $=$ & & & \\
\hline
\end{tabular}

Fuente: elaborado a partir de observaciones mencionadas en texto.

\subsection{Información política en época electoral}

La información política en época electoral ocupa de forma mayoritaria una posición subsidiaria en el conjunto de la información digital de las televisiones estudiadas: del total de 16.359 unidades analizadas, solamente 4.260 (lo que representa un $26,0 \%$ ) tiene que ver con la construcción estricta de la agenda mediática política o institucional. Los resultados arrojan diferencias sustanciales entre países y titularidad del medio (tabla 7).

De la muestra analizada, Argentina es el país con más información política desde el punto de vista porcentual (36,6\%) y Chile el que menos (11,30\%).

En cuanto a las correlaciones temáticas de las propias televisiones (tabla 8), existen similitudes estadísticamente re- 
levantes en los contenidos. Primero, la relación intra-país que se da de una forma intensa en Argentina $(0,671)$, Chile $(0,846)$ y Perú $(0,776)$ y de forma moderada en Ecuador y en Honduras. En segundo lugar, la correspondencia temática entre Televicentro (Honduras), Latina Televisión (Perú) y Ecuavisa (Ecuador), todas privadas $(0,818 ; 0,545 ; 0,755$, respectivamente) que no se da en las televisiones públicas de una forma tan sólida (correlación máxima de 0,671 entre Televisión Nacional de Honduras y Televisión Pública Argentina). Estos datos muestran que, a pesar de una tendencia general de construcción de la agenda con marcado carácter estatal, existente una transversalidad de ciertos temas en la dinámica regional que muestra coincidencias relevantes en televisiones públicas, pero, sobre todo, en las privadas (el sentido de la "democracia liberal" y el tratamiento de la política internacional).
Tabla 7. Unidades de información política, y porcentaje sobre el total de unidades analizadas en las 10 cadenas de televisión del estudio

\begin{tabular}{|l|l|c|c|}
\hline \multicolumn{1}{|c|}{ País } & \multicolumn{1}{|c|}{ Televisión } & N & $\%$ \\
\hline \multirow{3}{*}{ Argentina } & Televisión Pública Argentina & 931 & 45,3 \\
\cline { 2 - 4 } & Televisión Federal & 446 & 26,1 \\
\hline \multirow{2}{*}{ Perú } & TV Perú & 644 & 53,0 \\
\cline { 2 - 4 } & Latina Televisión & 334 & 12,7 \\
\hline \multirow{2}{*}{ Ecuador } & Ecuador TV & 492 & 32,3 \\
\cline { 2 - 4 } & Ecuavisa & 362 & 16,4 \\
\hline \multirow{2}{*}{ Honduras } & TNH & 200 & 28,3 \\
\cline { 2 - 4 } & Televicentro & 591 & 30,1 \\
\hline \multirow{2}{*}{ Chile } & TVN & 173 & 11,4 \\
\cline { 2 - 4 } & Canal 13 & 87 & 11,1 \\
\hline \multirow{2}{*}{ Total } & & & 26,0 \\
\hline \multirow{2}{*}{} & & & \\
\hline
\end{tabular}

Tabla 8. Correlaciones entre televisiones

\begin{tabular}{|c|c|c|c|c|c|c|c|c|c|c|}
\hline & TPA & $T L F$ & TVP & LAT & ETV & $E C U$ & TNH & $T L V$ & TVN & C13 \\
\hline$T P A$ & 1,000 & $0,671 * *$ & 0,378 & 0,441 & 0,490 & $0,853 * *$ & $0,671 * *$ & $0,713 * *$ & $0,531^{*}$ & $0,531^{*}$ \\
\hline$T L F$ & $0,671 *$ & 1,000 & 0,266 & 0,427 & 0,434 & $0,629^{*}$ & 0,483 & $0,608^{*}$ & $0,671 * *$ & $0,685^{* *}$ \\
\hline$T V P$ & 0,378 & 0,266 & 1,000 & $0,776 * *$ & 0,238 & 0,434 & 0,462 & $0,636^{*}$ & 0,189 & 0,336 \\
\hline$L A T$ & 0,441 & 0,427 & $0,776 * *$ & 1,000 & 0,147 & $0,545^{*}$ & 0,476 & $0,818^{* *}$ & 0,084 & 0,273 \\
\hline ETV & 0,490 & 0,434 & 0,238 & 0,147 & 1,000 & $0,629 *$ & 0,028 & 0,252 & $0,552^{*}$ & 0,315 \\
\hline$E C U$ & $0,853 * *$ & $0,629^{*}$ & 0,434 & $0,545^{*}$ & $0,629^{*}$ & 1,000 & 0,392 & $0,755^{* *}$ & $0,538^{*}$ & 0,392 \\
\hline TNH & $0,671 * *$ & 0,483 & 0,462 & 0,476 & 0,028 & 0,392 & 1,000 & $0,510^{*}$ & 0,455 & $0,545^{*}$ \\
\hline$T L V$ & $0,713^{* *}$ & $0,608^{*}$ & $0,636^{*}$ & $0,818^{* *}$ & 0,252 & $0,755^{* *}$ & $0,510^{*}$ & 1,000 & 0,420 & $0,615^{*}$ \\
\hline$T V N$ & $0,531^{*}$ & $0,671 * *$ & 0,189 & 0,084 & $0,552^{*}$ & $0,538^{*}$ & 0,455 & 0,420 & 1,000 & $0,846 * *$ \\
\hline C13 & $0,531^{*}$ & $0,685^{* *}$ & 0,336 & 0,273 & 0,315 & 0,392 & $0,545^{*}$ & $0,615^{*}$ & $0,846 * *$ & 1,000 \\
\hline
\end{tabular}

** La correlación es significativa al nivel 0,01 (unilateral).

* La correlación es significativa al nivel 0,05 (unilateral).

Fuente: elaborado a partir de observaciones mencionadas en el texto

\section{Discusión y conclusiones}

La agenda política digital de las televisiones latinoamericanas (analizadas en las elecciones presidenciales de Argentina, Perú, Ecuador, Honduras y Chile) promueve, con carácter general, una gran cantidad de noticias de infoentretenimiento, autopromoción y eventos que desplazan la información política a una posición subsidiaria que cuestiona, al menos en su plasmación en Twitter, la sobredimensión de este tipo de noticias en procesos electorales (verificación de h1). Este hecho se aprecia de una forma más marcada en las televisiones privadas, con una notable diferencia -en total- de más de 73 puntos porcentuales respecto a los entes públicos. Por ello, serían en mucha mayor medida las empresas informativas gubernamentales las que se estarían encargando de realizar el proceso de deliberación y socialización políticas para el conjunto de la ciudadanía. No obstante -y al margen de la titularidad del medio-, en todos y cada uno de los actores analizados el tema "Campaña"

Las televisiones públicas se sitúan con los candidatos oficialistas, y las televisiones privadas con la oposición, sin que la ideología sea un factor relevante

se sitúa como número 1 en rango, lo que refuerza algo que también se da en el campo analógico: primacía del horse-race campaign frente al issue-campaign. Esto implica el reforzamiento de un modelo de información política en medios de comunicación con mucha parafernalia y poca política, por lo que el soporte -o el medio- se pone al servicio del diálogo cruzado entre candidatos, al estar ausente la mediación y muy presente el llamado periodismo de declaraciones, que supone la primacía de la agenda política sobre la mediática.

La "coalición de agendas" o relación temática entre televisiones y candidatos genera áreas de impacto, rentabilidad electoral y determina la relación entre el sistema mediático y el político. Nuestro análisis ha evidenciado que existen aspectos reseñables al respecto en prácticamente todos los países analizados. La supeditación de la información mediática a la agenda política del candidato de turno es evidente en la Televisión Federal de Argentina (Macri), en Ecuador TV (Moreno), en Televicentro (Nasralla), Televisión Nacional de Honduras (Hernández) y en el caso chileno. La pauta es que 
las televisiones públicas se sitúan con los candidatos oficialistas, y las televisiones privadas con la oposición, sin que la ideología, en el segundo caso, sea un factor relevante (verificación de $\mathrm{H} 2$ ). A pesar de la relación, nuestra primera hipótesis principal $(\mathrm{H} 1)$-coalición de agendas mediáticas y las de candidatos oficialistas- queda refutada para dos de los casos, puesto que esa coincidencia no se produce en la Televisión Pública Argentina y en el caso chileno por existir una "coalición" a cuatro entre todas las televisiones y candidatos derivado de lo ya discutido en otros artículos precedentes (López-López; Vásquez-González, 2018): un sistema político de carácter centrípeto tiende a generar agendas comunes. No obstante, donde sí se muestra evidente la estrategia de cada ente es en el encuadre (análisis de atributos) y la frecuencia de aparición de cada uno de los candidatos. Por ello, el ente público de Ecuador, el público y el privado de Honduras, así como TFA de Argentina, se convierten en actores políticos que entran en la confrontación electoral y pierden su vocación de servicio público.

La tercera conclusión nace de la dificultad de establecer comparaciones temáticas entre las televisiones en América Latina, puesto que las particularidades de cada país determinan los temas a tratar. No obstante, cabe apuntar dos características relevantes desde el punto de vista investigador: así como hay una tendencia en los llamados países del Cono Sur a generar agendas entre candidatos con mayores niveles de correlación, esto también tiene su traslación mediática, ya que Chile y Argentina se sitúan en las primeras posiciones en cuanto a la relación temática en sus televisiones. En segundo lugar, sí existe una ligera tendencia en los medios comunicación privados a promover una agenda temática similar en el ámbito regional, con una presencia destacada del tratamiento de la política internacional y la institucionalidad (h2) lo que corroboraría una de nuestras hipótesis de partida.

Por todo ello se puede afirmar, con carácter general y en función de la pregunta de investigación formulada, que la información emitida en la Red por parte de las televisiones está aún caracterizada por el control político manifestado a través de mecanismos formales que pautan el propio ejercicio de la producción periodística y consagran la llamada "coalición de agendas". Contrastados estos resultados con otras investigaciones y comparada la agenda política y mediática digital en parte de un ciclo electoral en América Latina, es importante determinar en futuras investigaciones estos datos con las llamadas agendas sociales digitales, esto es, cómo interactúa, qué dice, qué piensa y cómo se relaciona el tercer agente que interviene en el espacio público en función de la definición clásica de comunicación política: la ciudadanía.

\section{Referencias}

Acinas-Vázquez, Juan-Claudio (2009). “Videopolítica y democracia”. Laguna: Revista de filosofía, n. 25, pp. 109-128.

Baggiolini, Luis; Castro-Rojas, Sebastián (2016). "Las redes de la política: Universo narrativo, campañas y microrrelato en Twitter". Inmediaciones de la comunicación, n. 11, pp. 159-180.

https://doi.org/10.18861/ic.2016.11.11.2621

Becerra, Martín (2014). Medios de comunicación: América Latina a contramano. Nueva sociedad, n. 249, pp. 61-74. https://nuso.org/articulo/medios-de-comunicacion-america-latina-a-contramano

Berrendo-Pérez, Óscar; Serrano-Fernández, Juan Antonio; Encinas-Puebla, Enrique (2019). Panorama audiovisual iberoamericano 2019. Informe Egeda.

https://bit.ly/2nz6rQ9

Cansino, César; Calles-Santillana, Jorge; Echeverría, Martín (2016). Del Homo Videns al Homo Twitter: democracia y redes sociales. Puebla, México: Benemérita Universidad Autónoma de Puebla. ISBN: 9786075252001

Cárdenas, Alejandro; Ballesteros, Carlos; Jara, René (2017). “Redes sociales y campañas electorales en Iberoamérica. Un análisis comparativo de los casos de España, México y Chile”. Cuadernos.info, n. 41, pp. 19-40. https://doi.org/10.7764/cdi.41.1259

Casero-Ripollés, Andreu (2009). "El control político de la información periodística”. Revista latina de comunicación social, n. 64, pp. 354-366.

https://doi.org/10.4185/RLCS-64-2009-828-354-366

Casero-Ripollés, Andreu (2015). "Estrategias y prácticas comunicativas del activismo político en las redes sociales en España". Historia y comunicación social, v. 20, n. 2, pp. 533-548.

https://doi.org/10.5209/rev_HICS.2015.v20.n2.51399

Castells, Manuel (2008). “Comunicación, poder y contrapoder en la sociedad red (I). Los medios y la política". Telos, 74, pp. 13-24. https://bit.ly/2o73dnn

Chadwick, Andrew (2013). The hybrid media system: Politics and power. New York: Oxford University Press. ISBN: 978 0199759477 
Chavero, Palmira; González, Juan-Jesús; Bouza, Fermín; Castromil, Antón; Rodríguez, Raquel (2013). “La mediatización de la agenda política: la discusión del pacto social como conflicto de agendas, 2009-2011". Revista latina de comunicación social, n. 68, pp. 639-655.

http://www.revistalatinacs.org/068/paper/994_Complutense/28_Palmira.html

Cohen, Jonathan; Tsfati, Yariv; Sheafer, Tamir (2008). "The influence of presumed media influence in politics: Do politicians' perceptions of media power matter?". Public opinion quarterly, v. 72, n. 2, pp. 331-344.

https://www.jstor.org/stable/25167628

https://doi.org/10.1093/poq/nfn014

De-Miguel, Juan-Carlos; Pozas, Víctor (2009). “¿Polarización ideológica o económica? Relaciones entre los medios y el poder político y corporativo". Viento Sur, n. 3, pp. 43-52.

http://cdn.vientosur.info/VScompletos/DeMigue\%20Pozas.pdf

Ekman, Mattias; Widholm, Andreas (2015). "Politicians as media producers: Current trajectories in the relation between journalists and politicians in the age of social media". Journalism practice, v. 9, n. 1, pp. 78-91.

https://doi.org/10.1080/17512786.2014.928467

Erro-Sala, Javier (2015). “Mediación tecnopolítica en el papel de los medios en una segunda transición democrática española”. En: Marco-Marco, Joaquín; Nicasio-Varea, Blanca. La regeneración del sistema, pp. 147-155. Valencia: Avapol. ISBN: 9788416063796

Fenoll-Tomé, Vicente; Cárcamo-Ulloa, Luis; Sáez-Trumper, Diego (2018). "El uso de Twitter de los medios de comunicación españoles en período electoral”. Estudios sobre el mensaje periodístico, v. 24, n. 2, pp. 1223-1238.

https://doi.org/10.5209/ESMP.62211

Fox, Elizabeth; Waisbord, Silvio (2002). Latin politics, global media. Texas: University of Texas Press. ISBN: 9780 292781894

Hallin, Daniel; Mancini, Paolo (2009). Sistemas mediáticos comparados: tres modelos de relación entre los medios de comunicación y la política. Madrid: Hacer Editorial. ISBN: 9788496913127

Larsson, Larsåke (2002). "Journalists and Politicians: a relationship requiring manoeuvring space". Journalism studies, v. 3, n. 1, pp. 21-33.

https://doi.org/10.1080/14616700120107310

López-Hermida-Russo, Alberto-Pedro; Vargas-Monardes, Josefina (2013). “La política relatada: el storytelling de Barack Obama en el marco de la Operación Gerónimo". Palabra clave, v. 16, n. 1, pp. 12-44.

https://dx.doi.org/10.5294/pacla.2013.16.1.1

López-López, Paulo-Carlos; Oñate, Pablo; Chavero-Ramírez, Palmira (2018). "El horse-race campaign frente al issue-campaign en la agenda digital: elecciones presidenciales en América Latina en el período 2015-2017". Revista ibérica de sistemas e tecnologias de informação, n. E16, pp. 270-282.

https://bit.ly/2ntLHsM

López-López, Paulo-Carlos; Romero-Ortega, Aldo-Benito; Ramos-Gil, Yalitza-Therly (2018). “La construcción del relato político a través de la narrativa transmedia: el caso del presidente Puigdemont en el proceso independentista catalán". Razón y palabra, v. 22, n. 2_101, pp. 144-167.

https://www.revistarazonypalabra.org/index.php/ryp/article/view/1194

López-López, Paulo-Carlos; Vásquez-González, Javier (2018). “Agenda temática y Twitter: elecciones presidenciales en América Latina durante el período 2015-2017". El profesional de la información, v. 27, n. 6, pp. 1204-1214.

https://doi.org/10.3145/epi.2018.nov.04

Martínez-Pandiani, Gustavo (2006). “El impacto de la televisión en la comunicación política moderna”. Signos universitarios, v. 25, n. 1, pp. 69-88.

https://p3.usal.edu.ar/index.php/signos/article/download/2955/3582

Mastrini, Guillermo; Becerra, Martín (2007). Presente y tendencias de la concentración de medios en América Latina. ZER: Revista de Estudios de Comunicación= Komunikazio Ikasketen Aldizkaria, v. 12, n. 22, pp. 15-40.

http://www.ehu.eus/ojs/index.php/Zer/article/download/3666/3298

McCombs, Maxwell E.; Shaw, Donald L. (1972). "The agenda-setting function of mass media”. Public opinion quarterly, v. 36, n. 2, pp. 176-187.

https://doi.org/10.1086/267990

McCombs, Maxwell E.; Shaw, Donald L.; Weaver, David H. (2014). "New directions in agenda setting theory and research". Mass communication and society, v. 17, n. 6, pp- 781-802.

https://doi.org/10.1080/15205436.2014.964871 
Noguera-Vivo, José Manuel (2010). "Redes sociales como paradigma periodístico. Medios españoles en Facebook". Revista latina de comunicación social, n. 65, pp. 176-186.

https://doi.org/10.4185/RLCS-65-2010-891-176-186

Pariser, Eli (2011). The filter bubble: What the Internet is hiding from you. London: Penguin Group. ISBN: 9781594203008

Rabadán, Pedro (2015). El sesgo en la televisión. La noticia política en campaña. Madrid: Editorial Universitas. ISBN: 978 8479914530

Rendueles, César; Sádaba, Igor (2014). “La hipótesis ciberpolítica: una aproximación crítica”. Documentación social, 173, pp. 95-116.

https://www.researchgate.net/publication/301286497_La_hipotesis_ciberpolitica

Salaverría, Ramón; Avilés, José-Alberto (2008). "La convergencia tecnológica en los medios de comunicación: retos para el periodismo". Trípodos, n. 23, pp. 31-47.

https://www.raco.cat/index.php/tripodos/article/viewFile/118910/154114

Sánchez-Galicia, Javier (2018). “La predisposición del voto en la era de la posverdad”. Más poder local, n. 35, pp. 36-40. https://dialnet.unirioja.es/descarga/articulo/6415344.pdf

Sartori, Giovani (1998). "La opinión teledirigida”. Claves de razón práctica, n. 79, pp. 2-7.

Scolari, Carlos (2008). Hipermediaciones: elementos para una teoría de la comunicación digital interactiva. Barcelona: Editorial Gedisa. ISBN: 9788497844109

Segado-Boj, Francisco; Díaz-Campo, Jesús; Lloves-Sobrado, Beatriz (2015): “Líderes latinoamericanos en Twitter. Viejas costumbres para nuevos medios en tiempos de crisis políticas". Revista latina de comunicación social, n. 70, pp. 156-173. https://doi.org/10.4185/RLCS-2015-1040

Soengas-Pérez, Xosé (2015). "El pluralismo y el control de la información en las televisiones generalistas españolas". Estudios sobre el mensaje periodístico, n. 21, pp. 199-207.

https://doi.org/10.5209/rev_ESMP.2015.v21.50675

Trejo-Delarbre, Raúl (1994). “¿Videopolítica vs. mediocracia? Los medios y la cultura democrática”. Revista mexicana de sociología, v. 56, n. 3, pp. 23-58.

https://doi.org/10.2307/3540847

Vu, Hong Tien; Guo, Lei; McCombs, Maxwell E. (2014). “Exploring the world outside and the pictures in our heads. A network agenda-setting study". Journalism \& mass communication quarterly, v. 91, n. 4, pp. 669-686. https://doi.org/10.1177/1077699014550090

Weaver, David H.; McCombs, Maxwell E.; Shaw, Donald L. (2004). "Agenda-setting research: Issues, attributes, and influences". In: Lee Kaid, Lynda. Handbook of political communication research, pp. 257-265. London: Lawrence Erlbaum Associates. ISBN: 9780805837759

Wolton, Dominique (1997). Penser la communication. Paris: Flammarion. ISBN: 9782080814135

\begin{tabular}{|c|c|c|c|}
\hline \multicolumn{2}{|c|}{ ÓXIMOS TEMAS } & \multicolumn{2}{|c|}{ http://www.elprofesionaldelainformacion.com/autores. } \\
\hline Número & Mes año & Tema & Envío textos \\
\hline 28,6 & Nov 2019 & Estructuras de poder en Comunicación & 10 jun 2019 \\
\hline 29,1 & Ene 2020 & Multidisciplinar & 10 oct 2019 \\
\hline 29,2 & Mar 2020 & Televisión: programas, audiencias y debates & 10 oct 2019 \\
\hline 29,3 & May 2020 & Relaciones públicas & 10 nov 2019 \\
\hline 29,4 & Jul 2020 & Investigación en Información y Comunicación & 10 ene 2020 \\
\hline 29,5 & Sept 2020 & Pluralismo informativo & 10 mar 2020 \\
\hline 29,6 & Nov 2020 & Framing (encuadre) & 10 may 2020 \\
\hline
\end{tabular}

